\title{
EDITORIAL
}

\section{Next-generation training}

\author{
Graduate training programmes must provide the tools and information necessary to help \\ trainees make informed decisions about their future career path, whether in science or not.
}

There has never been a more challenging time to begin a scientific career. For years, the number of Ph.D. candidates has grown unabated, but a corresponding increase in faculty positions has not been realized. Furthermore, the financial climate has made it increasingly difficult to access research funding, especially for those looking to start a laboratory. As a result, many young scientists now question whether a scientific career, either inside or outside of academia, is for them ${ }^{1,2}$. In the face of this disenfranchisement, it is prudent to ask whether the training that graduates receive allows them to make informed choices about whether to pursue a career in science, as well as how to succeed once that choice has been made.

Science has become increasingly interdisciplinary over the past 20 years. For instance, microbiologists now regularly use cellular, biochemical, molecular and genetic analyses together with bioinformatic, structural and computational approaches to address a problem. In this new environment, graduate students should be encouraged to become generalists rather than specialists, and to work in a range of disciplines. Many institutions already provide training programmes that include workshops to build on scientific skills such as statistics and bioinformatics, whereas other institutions go further and provide more technical training, for instance in particular experimental techniques or in computer programming. In the future, such programmes should perhaps be expanded to constitute a larger component of the overall training that graduates receive, so that they are better prepared for the varied demands of a scientific career. In the United Kingdom, in addition to such training programmes, collaborative Doctoral Training Partnerships (in which Ph.D. students are affiliated with two laboratories) are being introduced, with the aim of fostering a collaborative and interdisciplinary approach. Done well, such initiatives should expose graduate students to new ideas and provide inspiration for how to answer a scientific question using different approaches, and their introduction is to be encouraged.

Strange as it may sound, the scientific component may in fact not be the most important aspect of the graduate training process. The reality is that most $\mathrm{Ph} . \mathrm{D}$. students will not end up running an academic laboratory. Providing graduate students and early postdoctoral scientists with a realistic outlook on what a career in science entails, and what the alternatives are, is therefore essential.
The extent to which institutions currently provide such training varies dramatically worldwide. The United Kingdom seems to be fairly progressive in this regard, especially since the 2002 Roberts Report, which recommended that early-career researchers receive broad training in professional development. Graduate students and postdoctoral researchers at most UK institutions are now offered short courses in non-scientific skills and should have access to careers advice. However, even in the United Kingdom, challenges remain; the idea that academics need only provide training towards careers in academia remains entrenched in many supervisors, who can disengage from the mentoring process when a trainee reveals that they intend to leave academia. Furthermore, even in the best programmes, the breadth and depth of the professional development can be limited. Institutions should consider expanding the time and resources devoted to training programmes to include workshops on areas relevant to scientific and non-scientific careers alike, including management, marketing and economics.

Importantly, the next generation must be given a realistic view of what it takes to succeed in science so that they can make an informed choice about their future career path. In this regard, waiting until graduate school may be too late, and we should consider delivering courses on how science works' as part of undergraduate science degrees. Such courses could provide training in science communication, experimental design, ethics, publication, funding, public engagement and science history, as well as introducing the wide range of academic and non-academic careers that are open to science graduates. The introduction of such courses would help those entering science to better plan their career from the earliest stage and would have the added benefit of increasing the level of understanding of science and how it works among individuals who may go on to become leaders in areas such as business, economics, journalism and politics.

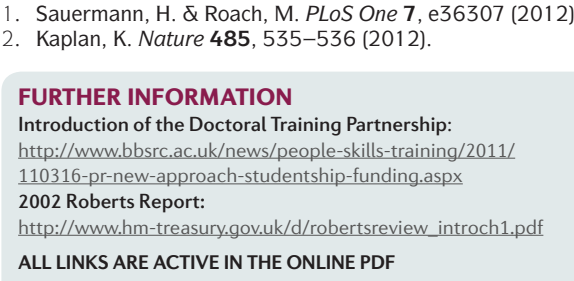

